\title{
Can Panel Data Methodologies Determine the Impact of Climate Change on Economic Growth? Richard A. Rosen *
}

\author{
Working Paper No. 171
}

\author{
November $17^{\text {th }}, 2021$
}

\begin{abstract}
Several major papers have been published over the last ten years claiming to have detected the impact of either annual variations in weather or climate change on the GDPs of most countries in the world using panel data-based statistical methodologies. These papers rely on various multivariate regression equations which include the annual average temperatures for most countries in the world as one or more of the independent variables, where the usual dependent variable is the change in annual GDP for each country from one year to the next year over 30-50 year time periods. Unfortunately, the quantitative estimates derived in these papers are misleading because the equations from which they are calculated are wrong. The major reason the resulting regression equations are wrong is because they do not include any of the appropriate and usual economic factors or variables which are likely to be able to explain changes in GDP/economic growth whether or not climate change has already impacted each country's economy. These equations, in short, exhibit suffer from "omitted variable bias," to use statistical terminology.
\end{abstract}

\section{https://doi.org/10.36687/inetwp171}

Keywords: climate change and economic growth, regression analysis, panel data methodologies, normal weather fluctuations vs. climate change, reforming peer review, omitted variable bias

JEL Classifications: A14, C1, C30, C33, Q51, Q56

\footnotetext{
* Richard A. Rosen received a Ph.D. in theoretical physics from Columbia University in 1974. After a post-doc at the Goddard Institute for Space Studies in New York, he began research on energy systems policy issues at the US Department of Energy's Brookhaven National Laboratory in 1976, and has continued that work ever since. He has testified in dozens of administrative law cases before numerous public utility commissions throughout the US on electric utility planning issues, and before US Federal Courts on Clean Air Act cases on behalf of the US Justice Department and state Attorneys General. He has been an official reviewer of several IPCC reports on climate change for the last several years.
} 


\section{Introduction}

Over the last ten years, a new sub-field of the economics of climate change has emerged, whereby several research groups have tried to use panel data for the GDPs of more than 100 countries or regions of the world extending over many decades to try to statistically quantify the impact that weather and/or climate change has had on those GDPs. There have been at least seven major papers published in this new sub-field, some of which primarily rely on the results of previous papers $(1,2,3,4,5,6$, and 7$)$. This work has gotten a fair amount of attention in both the news media and in climate change newsletters. (See, for example, the Proceedings of the National Academy of Sciences (3) and the April 22, 2015 New York Times (8).) In particular, some of these papers claim that as the average annual temperatures that countries experience over the next many decades increase due to climate change that these temperature increases will have a very large impact on GDP growth rates. These papers suggest that cooler countries will tend to have increases in their GDP growth rates due to climate change, and warmer countries will tend to have decreases in their growth rates.

Unfortunately, because the statistical methodologies relied on by each of these seven studies are not scientifically justified, both their quantitative and qualitative results are wrong and, I will argue, have seriously misled the climate change research community, policy makers, and the general public about many important issues. The key aspect of what is wrong with these statistical methodologies is that they violate the basic principles for the correct use of multivariate regression analysis for scientific research. This major methodological flaw is also potentially relevant to many other papers published in the social science literature, which is one rationale for writing this article. In addition, when attempting to determine the impact of changes in annual average temperature on changes in the GDP of individual countries, the papers discussed here (except one) do not even distinguish between the impact of larger but normal changes in the annual average weather from year to year, and the impact of the smaller and slower changes in weather from year-to-year due to climate change over a period of decades. (The exception, Kahn, et.al., still does not adequately disaggregate yearly weather from climate change (6).)

Some of these studies discussed here also claim that the likely impact on the GDPs of various countries and regions of various climate change scenarios can be calculated for the long-range future well beyond the time period covered by their database based on the past trends that they have computed, including as far into the future as the year 2100. Since many people are, naturally, extremely concerned about what impact climate change might have on their country's economy, and on the world economy in the future, these publications seem to offer interesting and important results that policy makers should take into account. Some of these papers have gotten a significant amount of attention in part, I believe, because they predict very large future impacts of climate change on most economies, both positive and negative, as noted above.

If the results of one or more of these papers were correct, they would support yet another argument in favor of mitigating climate change as fast as possible, namely to minimize negative economic impacts, for those countries and regions where negative impacts were projected to be likely. However, there are many other powerful arguments to support the rapid mitigation of climate change regardless of whether or not it negatively or positively impacts the GDP of countries. After all, the rebuilding of residential dwellings after devastating fires or storms 
caused by climate change might raise the GDP of the affected regions if such increases were deemed to be good, even though such a scenario is socially very undesirable. Thus, focusing on the impacts that climate change might have on national or regional GDPs is probably not the most fruitful approach to evaluating the negative impacts that climate change is likely to have on human societies and ecological systems, and is already having. Even from a strictly economic perspective, trying to deduce the likely impact of climate change on the total GDP of a country will not reveal important details as to the types of major economic harms that result, and how to prevent these harms. Presumably, the authors of the articles that are reviewed here would agree with this limitation inherent in their collective methodologies.

\section{Why are the seven major studies reviewed here wrong?}

As I will argue below, the results of all these studies discussed here are wrong for fairly simple but profound methodological reasons. The first reason is that the seven papers noted above do not utilize any economic theories and data for economic independent variables at all to help statistically explain the changes in the GDPs of countries or regions over decades, either before or after taking climate change into account. Therefore, the various multivariate regression equations that are utilized in these papers to try to detect the impact of changes in weather or climate (both temperature and precipitation) on changes in country GDPs from year to year do not include any of the usual candidates for economic, resource, or demographic variables that might explain (cause) such changes prior to trying to quantify the likely very small, incremental effect of climate change. Yet, there is a very extensive economics literature that has been published over decades that tries to explain economic growth via the use of such variables, which none of the papers reviewed here discuss. In fact, trying to explain GDP growth has been a major focus if not the major focus of the economics profession for a long time. (For example, see the famous paper by Robert Solow from the 1950s, and related research since then.(8))

Said slightly differently, the authors of the papers being reviewed here do not first try to statistically "explain" which of the usual economic, resource, and demographic factors seem to have the greatest causal impact on changes in the GDP of all the countries they include in their panel data assuming, first, that climate change did not yet exist such that fluctuations in annual weather were normal. In fact, the weather was mostly normal from 1960-78. It is only common sense that this analysis should be done first, because so far climate change is likely to have had a very small, if any, measurable effect on the GDP of countries even after 1978, except perhaps for countries where agricultural output constitutes a large fraction of their GDP. This is because agriculture is the most likely major industry to be strongly impacted by both normal weather and climate change. By definition, when doing good science in any given field of inquiry, one typically first analyzes the causes for the "first order" (the biggest) effects one is studying, and then one looks at the causal impact that "second order" (smaller) effects are likely to have on the first order effects. Here the first order effects are due to normal weather and the second order effects are due to climate change in each country.

Note that one must also consider the likely possibility that because GDP growth rates of many countries tended to fall over the 1960-2015 time period, and because climate change started to noticeably increase annual average temperatures for many of those same countries after 1978, that statistical findings interpreted by the authors of these papers that led them to believe that 
climate change caused lower GDP growth rates could have been simply due to coincidence (multicollinearity of those variables), and not due to a cause and effect relationship.

It is, therefore, well recognized as a basic principle of how to use multivariate regression analysis for scientific research that all (or most) independent variables that are theoretically relevant to explaining causal changes in the dependent variable must be included in the final regression equation, or at least tested for statistical significance and problems with multicollinearity as part of the research process. (See Donadelli 2017.(10)) If this is not done, as it was not in any of the papers reviewed here and important independent causal variables are omitted, all the coefficients derived for the other independent variables, such as temperature, are likely to be mis-estimated. This is the mathematical result of "omitted variable bias," as it is termed in statistics. In contrast to trying to analyze the potential causal impact of climate change on the GDP of a country as a whole, it might have made far more sense for these authors to have attempted to determine specific sectoral economic impacts in localized areas which have been directly and most severely affected by climate change (like agriculture). But it would have been difficult, probably impossible, to derive a long-run average for such economic impacts due to climate change over decades, and to project them into the future.

While in my opinion, this theoretical scientific critique of the seven papers being reviewed here based on sound scientific research methodology is sufficient to reject their qualitative and quantitative conclusions, I will provide a brief summary of some of the more specific methodological problems with a couple of the main regression equations relied upon in those papers. For example, the 2015 Burke, et.al., Letter in Nature entitled "Global non-linear effect of temperature on economic production" does not present the main regression equation on which it relies for its basic findings until deep into the Supplementary Materials, which I suspect most people who read the upfront Letter would not even read. Under the supplementary section "Empirical approach", their primary regression equation \#15 is first presented. Omitting its treatment of precipitation, the equation has a dummy independent variable for each country that applies in all years, a dummy variable for each year that applies to all countries, and a quadratic function of time specific to each country in the form $a t+b t^{2}$, where $t$ is time. This is why the authors claim to rely on a "standard" fixed effects methodology based on this approach to using dummy variables. Finally, there is a single quadratic function of temperature that, surprisingly, is the same for all countries and all years in the simple form again of $\mathrm{aT}+\mathrm{bT}^{2}$, where $\mathrm{T}$ is the annual average temperature for that country. Thus, there is not a single economic or demographic independent variable to be found in this equation, but the total number of coefficients listed above add up to hundreds of coefficients, mostly dummy variables, that need to be estimated by using the panel data that the authors have assembled. (Note also that none of the regression coefficients or statistics for these variables, except for the temperature variables, are included anywhere in the paper. Thus, the reader has no idea as to the goodness of fit that each regression equation has to the data, and, therefore, whether or not the equation makes any sense statistically.)

Not surprisingly, the authors claim that these hundreds of independent variables can capture the effects of whatever economic and demographic variables are relevant to explaining the historic changes in GDP which are used as the dependent variables in this regression equation for all 
years and countries. But they do not cite any economic theory or evidence supporting this hypothesis.

Of course, when a regression equation has hundreds of coefficients to be estimated from the panel data it should be clear that there is no theoretical or statistical basis for believing that any of the results has any causal significance in economic theory at all. With hundreds of coefficients almost any set of panel data could be "fit", the key question being how good is the fit. Furthermore, there is no basis for believing that such a "fixed effects" regression methodology is appropriate since there are obviously no fixed effects in this type of panel data. Nothing is "fixed" or constant or smoothly varying across all the countries or over time for all countries. The dummy variables for each country for all time are not appropriate because the GDP of each country changes very differently with respect to that for other countries over time, and the dummy variables for each year applicable to all countries are not appropriate because the GDPs of different countries grow by very different percentages in any given year. The GDPs do not grow in harmony with each other at all. The bottom line is, for example, if one had panel data like these authors all use for 100 countries over 40 years, 140 dummy variables could not begin to capture the complexity of the patterns of economic growth for these countries over time. There might actually be (100x39) or 3900 different growth rates involved for this set of panel data.

In addition, by focusing on the key function of temperature included in equation \#15, which after all is the main purpose of doing this entire analysis, we see that the quadratic function of temperature assumes that the economies of all countries respond to changes in temperature in exactly the same way in every year, since "a" and "b" are not country specific. Clearly, this cannot possibly be correct, and even in the remote possibility that it turned out to be true, it would have to be empirically demonstrated and not merely assumed, which these authors do not do. Anyway, it would be impossible to make this demonstration using a regression equation that included hundreds of coefficients that have no economic interpretation at all.

And do the authors really believe that economic growth for each country can properly be modeled assuming a quadratic function of time over the period of the database, with yearly adjustments made by the numerous dummy variables? One purpose of the statistical modeling of annual GDP growth is to determine how fast economic growth occurs over time, and not to assume that it varies over decades by a quadratic function of time, or any other specific functional form. Also, surprisingly, none of the values of the dummy variables nor the coefficients of the function of time are presented in the Supplementary Materials, or anywhere else in the papers under review, in order to allow the reader to get a feel for the possible realism of the results. And could equation \#15 with two quadratic terms, temperature and time, be at all useful for projecting the temperature effects out into the future for even 10 years, not to speak of out to 2100 as the authors do? Of course not; attempting to do so is absurd, in part because quadratic functions would tend to "blow up" as the key variables, time and temperature, increase beyond their values in the panel data.

To illustrate these methodological issues further, the research published by the various Burke, et.al., teams from 2015-2019 all utilizes the same annual time series panel data beginning in 1960 and ending in 2010, and the same regression equation (\#15) described above $(1,2,3)$. Their panel 
data includes country-specific annual average temperature data for this 50 -year period for 166 countries. However, one key fact that is not mentioned in their research papers, nor in any of the other publications discussed here, is that climate change was very weak over the period from 1960-1978, the first portion of their panel data. The average global temperature over land and water combined changed very little during this time period, with a slight cool spell in the mid1970s. Therefore, it is very unlikely that any statistical or causal methodology could possibly determine any net impact of climate change on the GDP of countries during this first 18 years of the database, since there was almost no climate change.

Thus, any serious attempt to discern the impact of climate change on economic systems of any type might do best to begin by analyzing economic and temperature data only after 1978 . This is because from 1978-2010, the second portion of their panel data, the average global temperature began to rise fairly steadily and to a much greater degree, increasing about 0.7 degrees $\mathrm{C}$ (Celsius) during this period. This includes temperatures over land and water. The cumulative increase from 1978, including the more recent years from 2010-2020, is about 1.1 degrees C. (The precise increase does not matter for the argument here, and the increase over land that would more directly affect country GDPs is generally somewhat greater than the increase over the oceans. (See IPCC 2018. (11))

In addition, one of the major methodological problems with the Burke, et.al. studies $(1,2,3)$, and the much newer Kalkuhl and Wenz paper (7), is that they do not even distinguish between changes in the weather, which Dell, et.al., reasonably defined as the year-to-year changes in annual average temperature (and precipitation) which exist whether or not climate change is occurring, and that component of temperature fluctuations and trends due to climate change. Climate change would, in theory, be represented in the temperature data by very slow multi-year increases that occur over a period of decades, but also by incremental annual fluctuations. This apparent conceptual confusion between these two key and fundamentally different aspects of temperature variability (annual average weather variability versus climate change) leads to temperature variables being used in the regression equations relied on by the Burke, et.al., and the Kalkuhl and Wenz research teams that do not take this distinction into account. Thus, only actual annual average temperatures that include both aspects of temperature variability are included in the regression equations derived in each study for each country or region, since that is all that can be derived from their panel data as it was constructed. No attempt was made by these authors to isolate or "pull out" of the yearly average temperature data a long-term climate change-based temperature component or trend. Thus, when Burke, et.al., tell their readers that they are projecting the impact of climate change on GDP in their various articles either historically, or out to the year 2100 , they are not really doing this. They are projecting results that are based on total annual temperature variations.

However, even though the Dell team distinguished these two components of their temperature time series conceptually, they did not discuss the feasibility of any practical analytical approach to achieve this key type of disaggregation. The only exception is in their 2014 paper where they briefly propose the possibility of dividing their 30 -year panel data into two 15 year periods, and averaging the temperature data over each 15 -year period separately (5). The problem with this approach, of course, is that climate change would, then, be represented by only one increase in 
temperature between the two 15-year averages, and the annual weather data would presumably no longer be used in a regression equation, which would surely not provide any basis for deriving statistically significant or interesting results for the separate impact on GDP of the two aspects of temperature - normal weather fluctuations versus climate change. This implies that neither of the methodologies relied on in the two Dell, et.al. papers $(4,5)$ would have been able to explicitly find any impacts on country GDPs due to climate change if this 15-year averaging methodology had been used, even if their statistical methodologies were otherwise scientifically valid, since there would be no variable in their regression equations representing climate change in a realistic way. The same is true of the newer Kahn, et. al., paper (6).

To be clear, what Dell, et. al. actually attempted to analyze in their papers was the impact of the total annual changes in temperature on the GDPs of a large number of countries. They did not claim to try to determine the separate impact of climate change. However, the Dell et.al. team simply assumed a simple production function for all country economies in all years, and then used this equation to try to fit hundreds of what they explicitly called country and time fixed effects (dummy variables). Again, including hundreds of dummy variables in a regression equation which attempts to explain changes in economic growth are not a substitute for an adequate economic theory of GDP growth. Even the use of their production function format for constructing their regression equation is completely unscientific, since Dell, et.al., did not demonstrate that such a function with its embedded dummy variables could reasonably model any of the country economies over time that were included in their panel data.

As noted above, common sense should tell any researcher that any climate change-based year to year impacts on country GDPs would be much smaller than the year-to-year changes in GDP caused by the total effect of major economic and demographic factors such as changes in interest rates, technologies for production, birth rates, education levels, inflation, trade, government investment, etc., factors that have been discussed in the economics literature on economic growth for decades. As the actual country-specific historical GDP data clearly shows, year-to-year changes in country-specific GDPs often range from minus several percent to plus several percent. In addition, country-specific GDPs often have average annual growth rates of plus 2.0-3.0 percent per year over fairly long periods of time, such as many decades. These changes in annual GDP co-exist with typical fluctuations in annual average temperatures of +/- 1.0-2.0 degrees Celsius for any given country.

In contrast, the average long-term change in temperature at the global level due to climate change, as noted above, was about 0.02 degrees $\mathrm{C}$ per year $(0.7$ degrees over the 32 years from about 1978-2010), with annual fluctuations due to climate change alone usually in the range of $+/-0.1$ degrees $C$, or less. Since the total annual fluctuations in temperature due primarily to changes in the normal weather are typically far greater (10-20 times greater) than the average annual increments in temperature due to climate change, one would expect that this would also be true of the relative impacts that these two causes of temperature change would usually have on the annual GDP of most countries. Thus, by relying on annual panel data for the GDP and temperatures of most countries in the world simultaneously, the likely impact of changes in temperature due to climate change on GDP would generally be swamped by the impact of normal (pre-climate change) annual temperature fluctuations. This poses a grave methodological 
problem for how the long-run cumulative impacts due to climate change could ever be estimated from panel data.

To repeat a point made above, the long-term slowdowns in economic growth for many high GDP Western countries over the last several decades probably had nothing to do with climate change. But to the statistical software such a trend would be collinear with a very slow increase in annual average temperatures due to climate change, leading to a small but negative coefficient for a linear temperature variable in a regression equation, even if no causality were involved. This is another purely statistical reason why it is probably impossible to use panel data to derive a valid causal relationship between temperature increases due to climate change and country GDPs.

While the above arguments lead me to strongly believe that year-to-year impacts of climate change on the GDP of a country would almost always be far smaller than the impacts of normal annual temperature fluctuations, this hypothesis could be wrong, of course. But, to my knowledge, no economist has ever challenged this assumption in a published scientific article. Again, this implies that the first task of any research effort to attempt to determine the relatively small past impact of climate change on country GDPs on an annual basis is to first statistically explain changes in those GDPs assuming that climate change did not exist.

To do this would require a very sophisticated theoretical and empirical research effort, which is not easy, to find the most significant economic, geographic, and demographic causal factors for changes in all country GDPs for the multi-decade period under analysis. The very interesting research paper by Cicconne and Jarociński 2010 (12) clearly demonstrates how difficult, if not impossible, this research effort would be if done correctly when relying on panel data for a large number of countries. But whether this research would be easy to do or not, none of the authors of the articles being discussed here, including the Dell, et.al., papers, included any of the usual kinds of economic factors listed in the Cicconne and Jarocinski paper as potential economic causal variables in the regression equations they estimated from the panel data, as noted above. They did not even discuss the serious problems with accurately measuring the GDP for different countries which use different currencies in the first place, as discussed by Cicconne and Jarocinski.

The implication of these omissions is that none of the research findings or regression equations in any of the papers discussed above have any scientific validity $(1,2,3,4,5,6$, and 7$)$. If one cannot statistically explain annual variations in the GDP of a large set of countries using panel data at a high level of confidence prior to the onset of significant climate change from 1960-1978, or prior to considering climate change in more recent data, surely one cannot hope to explain the more subtle and separate incremental impact on country GDPs of the modest amount of climate change that has occurred since 1978, or so. Using panel data that begins well before 1978, as these studies did, would also tend to wash out any climate change impact that could possibly be detected across the entire 50 or so year period, and that would be stronger after 1978. The obvious implication of this critique is that none of the seven journal articles mentioned above should have cleared the peer review process relied on by those journals. 


\section{Why the analysis of time series panel data will never likely result in scientifically justified results for the impact of climate change on a large group of country GDPs.}

What if one or more of the research teams, as discussed above, had attempted to "pull out" or disaggregate the annual temperature component due to climate change from the normal weather fluctuations for each country and each year for their time series panel data, and input the two different temperature variables that resulted into their statistical analysis of changes in GNP for those countries simultaneously? Would this have likely led to a statistically significant coefficient for the separate climate change temperature variable if the research teams had also first explained most of the variance of the annual GDPs of all countries simultaneously by key economic, geographical, and demographic factors? The answer is most probably "no".

One reason, of course, is that each country's GDP will respond to the same amount of temperature change both in the short and long-run somewhat differently from each other, because each economy has a somewhat different structure, different government policies, different amounts of air-conditioning and heating to respond to the weather, and different mixes of industries and agriculture in addition to different absolute temperature baselines. As noted above, Cicconne and Jarociński (12) demonstrate how difficult it is to find a stable set of statistically significant causal factors that explain GDP growth for all countries simultaneously using panel data, even once the usual candidates for causal factors are considered. They demonstrate that this is due, in part, to different economists calculating the annual GDP of each country using slightly different methodologies. Also, the relevance, then, of differences in the structure of the economies of different countries or regions has, unfortunately, been almost completely neglected in the research papers discussed here, except for brief mention of the relevance of the agricultural sector. The implicit assumption in the papers discussed here is that all countries would react similarly to the same changes in temperature both in the short and long-run, net of the impact of the "fixed effects" dummy variables that are not temperature dependent and that are included in the regression equations relied on in those papers.

Secondly, beyond consideration of structural differences in the economies of different countries, even climate scientists do not know how to decompose annual average time series temperature data for any single country into its "normal" value for each year, including normal annual variations, plus an additional component of change on an annual basis due solely to climate change. The problem is that both components of annual temperature vary almost randomly. Yet, being able to disaggregate annual temperature into these two different components is a necessary condition for being able to determine the impact of climate change by itself on economic growth. As the reader may know from numerous articles in the press and journals about climate change "attribution", it is difficult enough for climate scientists to be fairly sure as to whether or not any single storm was caused by or made more ferocious by climate change. But it is even more difficult to attribute heat waves and cold waves to climate change versus normal changes in the weather over a period of only a few weeks, which is how long hot and cold spells typically last. Thus, the annual impact of climate change on the annual temperature for any single country for a single year is impossible to determine accurately. Climate scientists can only determine the impact of climate change on temperatures for a given country over a multi-year period, for example over ten years. 
Thirdly, the impact of climate change could be represented at least in theory in a regression equation by a temperature variable that would vary annually but would also increase slowly but steadily over decades, as discussed previously. However, even if climate science advances to the point when this disaggregation between annual normal weather and climate change could be done fairly accurately, which cannot be done now, the slow increase over time that would characterize such a climate change variable would make it highly collinear with the steady but much faster increase or decrease in GDP on an annual percentage basis, as noted above. There would also likely be many other economic or demographic factors used as independent variables in trying to explain changes in GDP that would also tend to rise or fall slowly over time, on average, over a period of several decades. These relatively slow trends would introduce even more multicollinearity into the regression analysis between more independent variables. Thus, including a second temperature variable that varied slowly in time representing climate change into such a regression equation would not likely lend any significant causal explanatory power to the equation due to the presence of so much multicollinearity between so many variables. Therefore, its coefficient would not likely be significant at any interesting level, and, at best, its coefficient would be highly uncertain.

Furthermore, in order to establish convincing causal relationships between economic, demographic, or temperature variables and the GDP based on initially significant statistical findings for these coefficients, much additional analysis would be required in order to uncover the detailed microeconomic linkages and explicit economic causal mechanisms that would give theoretical support to any such statistical findings. In general, a finding of statistical significance for independent variables in a regression equation is not the end of a scientific inquiry, but rather only the middle of one, which the authors of the papers reviewed here also seem not to realize. My hypothesis and claim is that only if climate change were by far the dominant causal factor for changes in the GDP growth rates of all countries, which it clearly is not, could one hope to find a scientifically justified causal relationship between it and GDP growth using panel data for a large number of countries.

\section{How could climate change impacts on national or regional economies be studied?}

The most productive way in which to analyze the likely impact of climate change on the GDP of any country would be first to do a series of annual microeconomic studies on the economic consequences for that country or region of each major weather event that climate change appeared to influence. For example, considering the huge forest fires in California during the summer and fall of 2020, one could try to compare all the money spent in the short and long run to fight the fires and pay for all the other incremental costs society incurred due to the fires, to spending patterns that would have occurred if the additional fires caused by climate change had not occurred. Then, in the US, for example, a state-level macroeconomic model could be run for the two scenarios, the California economy with and without the extra fires occurring. Some fraction of the difference in economic impacts could, then, be attributed to climate change as opposed to normal weather conditions. If this economic difference also influenced the GDPs of other neighboring states within the US, this additional impact could be added in. Whatever the results of such a modeling effort would be, the impact of climate change on local economies would not be simply proportional to changes in a function of the annual country-wide 
temperature, as the papers discussed here explicitly assume. It is much more complicated than that, and the impact would be highly non-linear, and would depend on the kind of climate change event that occurred.

A more complete discussion of how past impacts of climate change on country or regional economies could be studied is beyond the scope of this critique. The key point is that such a set of calculations is probably best made for individual countries or sub-regions, where those aspects of climate change that have clearly affected and amplified specific weather events, and, thus, have incrementally and directly impacted the local economy, can be most accurately determined on a physical basis. Sweeping macro-economic methodologies such as applied to panel data attempting to analyze the impact of climate change on large groups of countries or regions simultaneously will probably never succeed in being sufficiently accurate or specific enough to ever be useful for policy makers. Anyway, it is already well known why the world needs act to mitigate climate change. The damage caused by climate change to people, social systems, economic systems, and ecological systems is quite apparent, whether or not it can be measured historically or predicted for the future in monetary terms, and it is clearly getting much worse over time.

\section{Why did the peer review process for this sub-field of research fail?}

Of course, as an independent reader of the journal articles discussed above, I cannot be sure why the major methodological issues and problems raised here were not readily apparent to the peer reviewers of these articles. The pitfalls in using multivariate regression analysis require careful attention. The issue goes well beyond simply disagreeing with a research team as to the best set of independent theoretically important variables to include in any particular regression analysis. These articles reflect a collective failure on the part of all of these authors to use any realistic economic theory and set of economic variables at all to explain variations in GDP, previously a very well-researched economic issue. Presumably, the authors were well aware of the fact that there is a vast economic literature extending over decades discussing the causes of economic growth.

In fact, Kalkohl and Wenz (7) were well aware of my specific criticisms of the similar research to theirs done by Burke, et.al., prior to publishing their paper, since they included my 2019 PNAS letter (13) in their list of references. Yet they did not seem to understand that my same basic criticisms also applied to their research methodology, as well as to the Burke, et.al., papers. Even more surprising is that the peer reviewers and editors at the PNAS published a seemingly important second paper that also relied on the Burke analysis in August 2021 after they had already published my 2019 critique of the Burke, et.al., papers (14). Thus, while the results regarding the likely impact of passing through tipping points on the GDP of the world as cited in the new PNAS paper were also publicized widely in the climate change-related media, they are also all wrong. The peer reviewers should have noticed these problems.(See Rosen 2015 for further critique of the peer review process.(15))

Beyond questioning the peer review process, it seems to me that teachers of economics, social science, and statistical analysis also need to ask themselves why so many young scholars made such egregious methodological mistakes. Any introductory textbook on multivariate regression 
analysis will state that as many theoretically important independent variables as possible should be included in any regression equation for the statistical significance of any independent variable to be taken seriously, and not be mis-estimated due to omitted variable bias.

The possible presence of multicollinearity is usually discussed as a problematic issue, which may limit the number and type of independent variables that should be included simultaneously. But how to deal with multicollinearity is a complex issue. Multivariate regression analysis is inherently incapable of estimating the coefficients of many different independent variables accurately when most of them change slowly over time, which may be the case when trying to determine the impact of several economic and demographic variables, plus a slowly varying climate change temperature variable, on changes in GDP. Multivariate regression analysis is, therefore, most accurate when both the independent and dependent variables vary substantially from one data point to another to different degrees so that multicollinearity would not likely be a significant problem.

\section{Conclusion}

The main focus of this review has been to identify how extremely simple but profound methodological errors or misunderstandings of sound scientific methodologies have led to the publication of a series of research papers that claim to have determined either the past impact of annual temperature variations or of climate change on the historical GDPs of a very large number of countries and regions over decades. Yet, as we have seen above, none of these papers has any scientific validity at all, and all their numerical results are wrong. These incorrect results have, presumably, already misled thousands of other researchers and potentially hundreds of thousands of members of the public who have either read the papers or have read media accounts about this research. Save for the PNAS letter I authored critiquing the most recent Burke, et. al., paper (13), I have not yet seen any other publicly available criticism of any of this research that would alert the public and policy makers to the problematic character of the research.

How can this have happened? How is it possible that no other scientists, especially the peer reviewers of these seven papers, have noticed that in each case the underlying methodology violated the standard norms of how multivariate regression analysis should be used for scientific research? Whatever the subject matter is, for a regression equation to be potentially useful for determining causes and effects, it must include most if not all the independent variables deemed to be potentially causative factors to explain changes in the dependent variable. This critique, then, provides a warning to researchers in the many fields of social science that rely heavily on multivariate regression analysis. Be careful not to proceed with statistical analysis without a sound causal theory of the phenomena being researched.

What must be done now to try to prevent such a situation from arising in other fields of social scientific research? In my judgement, one necessary reform is that the peer reviews of all pending scientific articles should be made public in the relevant journal before any article is finally published. Other members of each scientific community should be given a chance to review the peer reviews to determine if the original set of peer reviews are themselves rigorous. Only once the journal editors have a chance to get feedback from the broader scientific 
community as to the adequacy of the peer reviews, should they rely on them for providing feedback to the authors of the relevant article, and for publication decisions.

This proposed reform may slow down the rate of new publications somewhat, but it is clearly much better for the research community that journals publish high quality articles somewhat more slowly, than bad articles quickly. This proposed reform process will also probably lead to better peer reviews if the reviewers know that the content of their reviews will be made public, even if their names are not. Finally, if even using such a reformed process for peer reviews still leads to unscientific articles being published, journal editors should be much more receptive to arguments from independent scientists as to why individual articles should be retracted or corrected in print. While retracting articles should be a last resort, it is definitely required at times, as recent history in many fields of science has demonstrated.

\section{Afterword}

Surprisingly, after the basic analysis of the paper reviewed here was finalized as described above, a new and very elaborate set of statistical analyses by Newell, et.al., entitled "The GDPTemperature Relationship: Implications for Climate Change Damages" appeared as a preprint (16). The authors of this new paper did approximately 800 regression analyses of a similar nature to those done by the Dell and Burke research teams, in effect checking the reasonableness of their work. Newell, et.al., stated the purpose and conclusion in doing this vast amount of work as: "By estimating 800 plausible specifications of the temperature-GDP relationship, we demonstrate that a wide variety of models are statistically indistinguishable in their out-of-sample performance, including models that exclude any temperature effect." They continued: "Yet estimation of the scope and magnitude of climate impacts on the economy is hindered by the temporal invariance of climate over relevant time frames." This is a similar way of stating the fact noted earlier that climate change was weak or non-existent in the temperature data from about 1960-1978, which overlaps with the time period studied by the papers under review here.

Then Newell, et.al., make some new and important observations about the difficulties in trying to econometrically determine the causal impact of climate change on GDP, and these observations complement the critique above. They say: "Moreover, the econometric approach confronts two challenges in estimating aggregate economic impacts of climate change. First, identification of climate effects from weather variation requires strong assumptions about dynamic processes like adaptation and the persistence of idiosyncratic temperature responses amid secular climate change." "Second, theory [as used by the authors of the papers under review here] does not prescribe specific, estimable, structural relationships between climate and economic outcomes." Obviously, they are making the same point as our fundamental criticism of the lack of a theoretical basis for the regression equations analyzed, as discussed at length above. Finally, Newell, et.al., conclude that "accounting for uncertainty among models of superior performance in our estimation procedure, we find that the marginal effect of temperature on GDP growth $i s$ not distinguishable from zero [emphasis added] at annual average temperatures observed in our data." Thus, it should be clear that this huge and elaborate statistical analysis performed by Newell's team confirms the basic hypothesis of this paper, namely that it is extremely unlikely that the impact of climate change on specific country GDPs can ever be statistically determined via the use of panel data of the type used in all the research papers reviewed here $(1,2,3,4,5,6$, and 
7). The main contribution of this paper is to explain the theoretical basis for why the elaborate and seemingly definitive Newell, et.al., results were inevitable. 


\section{Annotated References}

1. Burke, M., Hsiang, S., and Miguel, E., Global non-linear effect of temperature on economic production, Nature, 527, 235-239, 2015. https://doi.org/10.1038/nature15725. This paper presented the basic statistical analysis of the impact of temperature and precipitation on the GDP of most countries in the world using panel data.

2. Burke, M., Davis, W.M., and Diffenbaugh, N.S., Large potential reduction in economic damages under UN mitigation targets, Nature, 553, 549-553, 2018.

https://doi.org/10.1038/s41586-018-0071-9. This paper relied on the statistical analysis presented in the Burke, et.al., 2015 paper.

3. Diffenbaugh, N.S. and Burke, M., Global warming has increased global economic inequality, Proceedings of the National Academy of Sciences, April 2019. https://doi.org/10.1073/pnas.1816020116. This paper also relied on the statistical analysis presented in the Burke, et.al., 2015 paper.

4. Dell, M., Jones, B.F., and Olken, B.A., Temperature Shocks and Economic Growth: Evidence from the Last Half Century, American Economic Journal, 4(3), 66-95, 2012. https://doi.org/10.1257/mac.4.3.66. This describes the authors attempt to analyze the impact of temperature changes on the GDP of most countries in the world.

5. Dell, M., Jones, B.F., and Olken, B.A., What do we learn from the weather? The new climate-economy literature, Journal of Economic Literature, 52(3), 740-798, 2014. https://DOI: 10.1257/jel.52.3.740. This is a review paper for much of the literature as of 2014 on temperature impacts on various aspects of country economies, but not climate change explicitly.

6. Kahn, M.E., Mohaddes, K., Ng, R., Pesaran, M., Raissi, M. and Yang, J., Long-Term Macroeconomic Effects of Climate Change: A Cross-Country Analysis, International Monetary Fund Working Paper, 2019. This is a statistical analysis of an even larger set of panel data for the GDP for over 1000 regions of the world.

7. Kalkuhl, M. and Wenz, L., The impact of climate change on economic production. Evidence from a global panel of regions, Journal of Environmental Economics and Management, 103, 2020. https://doi.org/10.1016/j.jeem.2020.102360. This paper differs in part from the 2015 Burke, et.al. analysis by relying on panel data for more than 1000 regions of the world. How they derive such detailed panel data is not properly described in either the paper or the supplementary materials, but this is not of central importance to the critique presented here.

8. Sengupta, S., Global Wealth Gap Would Be Smaller Today Without Climate Change, Study Finds, New York Times, April 22, 2019.

9. Solow, R.M., A Contribution to the Theory of Economic Growth, The Quarterly Journal of Economics, 70, no. 1, 65-94, February 1956. This article is considered by many to be a seminal paper on the theory of economic growth, but there are many others.

10. Donadelli, M., Jüppner, M., Riedel, M., and Schlag, C., Temperature shocks and welfare costs, Journal of Economic Dynamics and Control, 82, 331-355, 2017. https://DOI: 10.1016/j.jedc.2017.07.003. This paper included macroeconomic variables along with temperature in a regression equation for only the US. Therefore, theoretically, this is a possible start for a logically reasonable methodology to study temperature shocks, but 
since only one country is treated it is not based on panel data, and it only attempts to determine the impact of annual weather variations in total on the US GDP, and not climate change separately.

11. IPCC Special Report on 1.5 Degree Celsius Scenarios, October 2018. This report provides basic data on the time trend of temperature changes due to climate change. Also, see New York Times, January 9, 2021 for updated data.

12. Ciccone, A. and Jarociński, M., Determinants of Economic Growth: Will Data Tell?, American Economic Journal: Macroeconomics , 2(4), October, 222-246, 2010. https://DOI: 10.1257/mac.2.4.222. This article shows the problems associated with trying to find statistically significant economic factors that cause GDP growth given the problems with accurately measuring GDP.

13. Rosen, R.A., Temperature impact on GDP growth is overestimated, Proceedings of the National Academy of Sciences, August 2019. https://DOI: 10.1073/pnas.1908081116. This letter is a critique of the Diffenbaugh, et. al., 2019 paper also published in the PNAS, and the Burke, et.al., analysis on which it relies.

14. Dietz, S. et.al., Economic impacts of tipping points in the climate system, PNAS, 118 (34), online August 24, 2021. This paper tries to calculate for each of eight well-known potential tipping points in the earth-climate system what the impact on the global GDP might be once the tipping point has been passed. The final step in their analysis relies solely on the Burke economic analysis from 2015 in relating changes in GDP over the next many decades to the changes in temperature that the authors believe will be caused by each tipping point being triggered.

15. Rosen, R.A., IAMs and peer review, Nature Climate Change 5, 390, 2015. This letter discusses some of the problems with the peer review process, especially when research articles need to be reviewed which are based on large quantities of data and complex models which are not documented properly in the papers submitted for publication.

16. Newell, R.G., Prest, B.C., Sexton, S.E., The GDP-Temperature relationship: Implications for climate change damages, Journal of Environmental Economics and Management (21), https://doi.org/10.1016/j.jeem.2021.102445. This paper performs 800 regression analyses of the panel data as used by the Dell and Burke teams in their various papers to demonstrate that the impact of climate change/temperature on the GDPs of the set of over 150 countries included cannot be determined to be statistically significant. 